\title{
Some estimates for resolvent operators under the discretization by finite element method
}

\author{
R. Figueroa-López · G. Lozada-Cruz
}

Received: 25 November 2013 / Revised: 3 July 2014 / Accepted: 11 July 2014 /

Published online: 31 July 2014

(C) SBMAC - Sociedade Brasileira de Matemática Aplicada e Computacional 2014

\begin{abstract}
This paper is devoted to obtain some norm estimates for the difference between the two resolvent operators under the discretization of the domain $\Omega \subset \mathbb{R}^{n}, n \geqslant 2$, by finite element method.
\end{abstract}

Keywords Parabolic equations · Finite elements method $\cdot$ Resolvent operator approximation

Mathematics Subject Classification $\quad 35 \mathrm{~K} 15 \cdot 65 \mathrm{M} 60 \cdot 47 \mathrm{~A} 58$

\section{Introduction and statement of the main result}

Let $\Omega \subset \mathbb{R}^{n}, n \geqslant 2$, be a bounded domain, and assume either that the boundary $\partial \Omega$ is smooth or that $\Omega$ is a polyhedral domain. Let the second order uniformly strongly elliptic operator be given by

$$
L=\sum_{i, j=1}^{n} a_{i j}(\cdot) \frac{\partial^{2}}{\partial x_{i} \partial x_{j}}+\sum_{j=1}^{n} b_{j}(\cdot) \frac{\partial}{\partial x_{j}}+(c(\cdot)+\lambda)
$$

where the coefficients $a_{i j}, b_{j}, c: \bar{\Omega} \rightarrow \mathbb{R}, a_{i j}=a_{j i}, i, j=1, \ldots, n$ are smooth functions and $\lambda$ be a parameter to be specified later.

Communicated by Ruben Spies.

R. Figueroa-López $(\varangle) \cdot$ G. Lozada-Cruz

Department of Mathematics, Universidade Estadual Paulista (UNESP), Rua Cristóvão Colombo, 2265, São José do Rio Preto, SP CEP 15054-000, Brazil

e-mail: rodiak@ibilce.unesp.br

G. Lozada-Cruz

e-mail: german@ibilce.unesp.br 
Consider the initial boundary value problem of parabolic type

$$
\left\{\begin{array}{c}
u_{t}=L u, \quad t>0, \quad x \in \Omega \\
u=0, \quad t>0, \quad x \in \partial \Omega, \\
u(x, 0)=\varphi(x), \quad x \in \Omega
\end{array}\right.
$$

where $\varphi \in H_{0}^{1}(\Omega)$.

Let $X=L^{2}(\Omega)$ be a Hilbert space and consider the nonselfadjoint linear operator $A$ : $D(A) \subset X \rightarrow X$ by

$$
\begin{aligned}
D(A) & =H^{2}(\Omega) \cap H_{0}^{1}(\Omega), \\
A u & =-L u, \quad \forall u \in D(A) .
\end{aligned}
$$

In Figueroa-López and Lozada-Cruz (2014) was shown that operator $A$ is sectorial in $X$ and assuming that $\lambda$ is chosen such that $\operatorname{Re} \sigma(A)>0$, we can define the fractional powers $A^{\alpha}$ and the corresponding fractional power spaces $X^{\alpha}:=D\left(A^{\alpha}\right), \alpha>0$, endowed with the graph norm (see Cholewa and Dlotko 2000, Section 1.3.3). $X^{\alpha}$ is a Hilbert space with the inner product $\langle\phi, \psi\rangle_{\alpha}=\int_{\Omega} A^{\alpha} \phi A^{\alpha} \psi$. Then, $X^{1}=D(A), X^{0}=L^{2}(\Omega)$ and $X^{1 / 2}=H_{0}^{1}(\Omega)$.

As usual the problem (1) can be written as an abstract evolution equation in $X$

$$
\left\{\begin{array}{l}
\dot{u}+A u=0, \quad t>0, \\
u(0)=\varphi \in X^{1 / 2}
\end{array}\right.
$$

Fujita-Mizutani (see Fujita and Mizutani 1976) made a operator theoretical study of the finite element method applied to the initial boundary value problems for partial differential equations of parabolic type (1).

The discretization of the problem (AP) using the finite element method has the form

$$
\left\{\begin{array}{l}
\dot{u}_{h}+A_{h} u_{h}=0, \\
u_{h}(0)=\varphi_{h} \in X_{h}^{1 / 2}
\end{array}\right.
$$

where $X_{h}^{1 / 2} \subset X^{1 / 2} \cap C(\Omega)$ is a finite dimensional space obtained from the discretization of domain $\Omega$ by the finite element method, $h$ is the largest diameter of each subdivision of the domain and $A_{h}: X_{h}^{1 / 2} \rightarrow X_{h}^{1 / 2}$ is the discretization of the operator A. More details on $A_{h}$ and $X_{h}^{1 / 2}$ are given in the next section.

Let $u=u(t, \varphi)$ be the solution of the problem (AP) and let $u_{h}=u_{h}\left(t, \varphi_{h}\right)$ be the solution of the problem (1). In this context, it is natural to ask if $h$ approaching to zero implies $u_{h}$ to approximate $u$. To answer this question, we need to compare the solutions $u$ and $u_{h}$ in a certain sense when $h$ tends to zero. We can see that $u$ and $u_{h}$ are living in different spaces.

Using the integral representation(the Dunford-Taylor integral), we have

$$
u(t, \varphi)=\mathrm{e}^{-t A} \varphi=\frac{1}{2 \pi i} \int_{\Gamma_{1}} \mathrm{e}^{-z t}(z-A)^{-1} \varphi \mathrm{d} z,
$$

where $\Gamma_{1}$ is the positively oriented boundary, running from $\infty \mathrm{e}^{-i \theta_{1}}$ to $\infty \mathrm{e}^{i \theta_{1}}$, of the sector $\Sigma_{1}=\left\{z \in \mathbb{C}:|\arg (z)|<\theta_{1}\right\}$ (see Pazy 1992, Theorem 1.7.7). Also, for $u_{h}$, we have a similar integral representation. With this, we can see that to compare $u$ and $u_{h}$, we need to compare the resolvent operators $(z-A)^{-1}$ and $\left(z-A_{h}\right)^{-1}$ of $A$ and $A_{h}$, respectively, i.e., we will show that $\left(z-A_{h}\right)^{-1}$ converge in an appropriate way to $(z-A)^{-1}$. This is a key point to our work. With the convergence of the resolvent operators $A_{h}^{-1}$ to $A$ one can show, with a Trotter-Kato-type formula, the convergence of the linear semigroups $\mathrm{e}^{-t A_{h}}$ to $\mathrm{e}^{t A}$. This 
analysis has been proved to be successful when addressing to the behavior of the long-time dynamics in different perturbation problems (see Arrieta et al. 2006; Carvalho et al. 2013).

Now, we are in a position to formulate our main result.

Theorem 1 If Assumption 1 holds, then, there exists a positive constant $C$ and an acute angle $\theta_{1}$ such that for any $f \in X$ and $z \in \mathcal{S}_{0, \theta_{1}}$, we have

$$
\begin{aligned}
\left\|(z-A)^{-1} f-\left(z-A_{h}\right)^{-1} P_{h} f\right\|_{X^{1 / 2}} & \leqslant C h\|f\|_{X}, \\
\left\|(z-A)^{-1} f-\left(z-A_{h}\right)^{-1} P_{h} f\right\|_{X} & \leqslant C h^{2}\|f\|_{X}, \\
\left\|(z-A)^{-1} f-\left(z-A_{h}\right)^{-1} P_{h} f\right\|_{X} & \leqslant C h|z|^{-1 / 2}\|f\|_{X},
\end{aligned}
$$

where $\mathcal{S}_{0, \theta_{1}}=\left\{z \in \mathbb{C}: \theta_{1} \leqslant|\arg (z)| \leqslant \pi, z \neq 0\right\} \subset \rho(A)$ and $P_{h}$ is projection operator from $X$ to $X_{h}^{1 / 2}$.

Theorem 1 was proved by Fujita-Mizutani (Fujita and Mizutani 1976, Theorem 3.1) and Fujita-Saito-Suzuki (Fujita et al. 2001) for a bounded domain $\Omega \subset \mathbb{R}^{2}$ with smooth boundary or $\Omega$ a convex polygon.

The main goal of this paper is to show that Theorem 1 remains valid when $\Omega \subset \mathbb{R}^{n}$ is a bounded domain or a polyhedral domain with $n>2$, which has $\left\{\mathcal{T}^{h}\right\}_{h \in(0,1]}$ a quasi-uniform family of subdivisions and a reference element $(K, \mathcal{P}, \mathcal{N})$ of class $C^{0}$ with $K$ a "star-shaped" domain with respect to some ball. More details are given in Sect. 2.

Note that the norm estimates for the resolvent established in Gil' $(2012,2013)$ allow us to obtain the concrete bounds for the resolvent of $A$, when it is nonselfadjoint.

This paper is organized as follows. In Sect. 2, we introduce some notations and provide the discretization of the domain $\Omega$ needed to solve our problem. In Sect. 3, we obtain the rate of convergence of the resolvents and proceed with the proof of our main result.

\section{Notations and preliminaries}

In this section, we introduce some notations and we give the discretization of the domain $\Omega \subset \mathbb{R}^{n}$ with $n \geqslant 2$.

Since $A$ is sectorial, we can associate it with a sesquilinear form $\sigma: X^{1 / 2} \times X^{1 / 2} \rightarrow \mathbb{C}$ given by

$$
\begin{array}{r}
\sigma(u, v)=\langle A u, v\rangle_{X}, \quad u \in D(A), v \in X^{1 / 2}, \\
|\sigma(u, v)| \leqslant c_{1}\|u\|_{X^{1 / 2}}\|v\|_{X^{1 / 2}}, \quad u, v \in X^{1 / 2} \\
\operatorname{Re} \sigma(u, u) \geqslant c_{2}\|u\|_{X^{1 / 2}}^{2}-\delta\|u\|_{X}^{2}, \quad u \in X^{1 / 2},
\end{array}
$$

where the constants $c_{1}, c_{2}$ are positives and $\delta<\infty$ (see Brenner and Scott 1996, Section 5.6). In particular if $\delta=0$, we have

$$
\operatorname{Re} \sigma(u, u) \geqslant c_{2}\|u\|_{X^{1 / 2}}^{2} .
$$

Also, there are positive constants $\theta_{1}$ and $M_{1}$ with $\theta_{1}<\pi / 2$ such that

$$
\begin{gathered}
\mathcal{S}_{0, \theta_{1}}=\left\{z \in \mathbb{C}: \theta_{1} \leqslant|\arg (z)| \leqslant \pi, z \neq 0\right\} \subset \rho(A) \text { and } \\
\left\|(z-A)^{-1}\right\| \mathscr{L}(X) \leqslant \frac{M_{1}}{|z|}, \quad \forall z \in \mathcal{S}_{0, \theta_{1}} .
\end{gathered}
$$

Furthermore, a solution of the initial value problem (AP) is given by $u(t, \varphi)=\mathrm{e}^{-t A} \varphi$, $t \geqslant 0$, where $\left\{\mathrm{e}^{-t A}: t \geqslant 0\right\}$ is the analytic semigroup in $X^{1 / 2}$ generated by $-A$ (see Cholewa and Dlotko 2000, p. 35; Henry 1981, p. 21). 
Now, we describe the discretization of the domain $\Omega \subset \mathbb{R}^{n}$ with $n \geqslant 2$, using the finite element method. Thus, we will be able to discretize the problem (AP) and study the limitations given in Fujita and Mizutani (1976) using the theory made in Brenner and Scott (1996) for its generalization to $n>2$.

For a better understanding of the work and for convenience of the reader, we recall some definitions and results of Brenner and Scott (1996, Chapters 3 and 4).

\section{Definition 1 Let}

(i) $K \subseteq \mathbb{R}^{n}$ be a bounded closed set with nonempty interior and piecewise smooth boundary (the element domain),

(ii) $\mathcal{P}$ be a finite-dimensional space of functions on $K$ (the space of shape functions) and

(iii) $\mathcal{N}=\left\{N_{1}, N_{2}, \ldots, N_{k}\right\}$ be a basis for $\mathcal{P}^{\prime}$ (the set of nodal variables).

The triple $(K, \mathcal{P}, \mathcal{N})$ is called a finite element.

Definition 2 Given a finite element $(K, \mathcal{P}, \mathcal{N})$, let the set $\left\{\phi_{i}: 1 \leqslant i \leqslant k\right\} \subseteq \mathcal{P}$ be the basis dual to $\mathcal{N}$. If $v$ is a function for which all $N_{i} \in \mathcal{N}, i=1, \ldots, k$, are defined, then we define the local interpolant by

$$
\mathcal{I}_{K} v:=\sum_{i=1}^{k} N_{i}(v) \phi_{i} .
$$

Proposition 1 Let $\mathcal{I}_{K}$ be the local interpolant, then

(i) $\mathcal{I}_{K}$ is linear.

(ii) $N_{i}\left(\mathcal{I}_{K} v\right)=N_{i}(v), i=1, \ldots, k$.

(iii) $\mathcal{I}_{K}(v)=v$ for $v \in \mathcal{P}$. In particular, $\mathcal{I}_{K}$ is idempotent, i.e., $\mathcal{I}_{K}^{2}=\mathcal{I}_{K}$.

Proof See Propositions 3.3.4, 3.3.5 and 3.3.7 in Brenner and Scott (1996).

Definition 3 A subdivision of the domain $\Omega \subset \mathbb{R}^{n}$ is a finite collection of element domains $\left\{K_{i}\right\}_{i \in \mathbb{N}}$ such that int $K_{i} \cap$ int $K_{j}=\emptyset$ if $i \neq j$ and $\bigcup_{i \in \mathbb{N}} K_{i}=\bar{\Omega}$.

Definition 4 Let $(K, \mathcal{P}, \mathcal{N})$ be a finite element with $K \subset \Omega \subset \mathbb{R}^{n}$ and let $F: \mathbb{R}^{n} \rightarrow \mathbb{R}^{n}$ be an affine map given by $F(x)=\mathbf{A} x+\mathbf{b}$ where $\mathbf{A}$ is a nonsingular matrix and $\mathbf{b}$ is a nonzero vector in $\mathbb{R}^{n}$. The finite element $(\widehat{K}, \widehat{\mathcal{P}}, \widehat{\mathcal{N}})$ is affine equivalent to $(K, \mathcal{P}, \mathcal{N})$ if $F(K)=\widehat{K}$, $F^{*} \widehat{\mathcal{P}}=\mathcal{P}$ and $F_{*} \mathcal{N}=\widehat{\mathcal{N}}$, where $F^{*}$ is the "pull-back" of $F$ defined by $F^{*}(\hat{f}):=\hat{f} \circ F$, and $F_{*}$ is the "push-forward" of $F$ defined by $\left(F_{*} N\right)(\hat{f}):=N\left(F^{*}(\hat{f})\right)=N(\hat{f} \circ F)$.

Definition 5 The domain $\Omega$ is star-shaped with respect to ball $B$ if, for all $x \in \Omega$, the closed convex hull of $\{x\} \cup B$ is a subset of $\Omega$. The domain $\Omega$ is star-shaped with respect to some ball if there is a ball $B$ such that $\Omega$ is star-shaped with respect to ball $B$.

For example, any convex domain $\Omega \subset \mathbb{R}^{n}$ is star-shaped with respect to each ball $B \subset \Omega$.

Lemma 1 If a bounded domain $\Omega \subset \mathbb{R}^{n}$ is start-shaped with respect to a ball $B \subset \Omega$, then it satisfies the cone condition and has a Lipschitz boundary with the parameters depending only on $\operatorname{diam}(B), \operatorname{diam}(\Omega)$ and $n$.

Proof See Lemmas 3.2.3 and 4.3.5 in Burenkov (1998).

Definition 6 Let $\Omega \subset \mathbb{R}^{n}$ be a given domain and let $\left\{\mathcal{T}^{h}\right\}_{h \in(0,1]}$ be a family of subdivisions such that

$$
\max \left\{\operatorname{diam}(T): T \in \mathcal{T}^{h}\right\} \leqslant h \operatorname{diam}(\Omega) .
$$


(i) The family $\left\{\mathcal{T}^{h}\right\}_{h \in(0,1]}$ is said to be quasi-uniform if there exists $\rho>0$ such that

$$
\min \left\{\operatorname{diam}\left(B_{T}\right): T \in \mathcal{T}^{h}\right\} \geqslant \rho h \operatorname{diam}(\Omega), \quad \forall h \in(0,1],
$$

where $B_{T}$ is the largest ball contained in $T$ such that $T$ is star-shaped with respect to $B_{T}$.

(ii) The family $\left\{\mathcal{T}^{h}\right\}_{h \in(0,1]}$ is said to be non-degenerate or regular if there exists $\rho>0$ such that for all $T \in \mathcal{T}^{h}$ and for all $h \in(0,1]$,

$$
\operatorname{diam}\left(B_{T}\right) \geqslant \rho \operatorname{diam}(T) .
$$

Remark 1 If a family is quasi-uniform, then it is non-degenerate, but not conversely.

Definition 7 A reference element $(K, \mathcal{P}, \mathcal{N})$ is said to be a $C^{r}$ element if $r$ is the largest non-negative integer for which the finite element space satisfies

$$
V^{h}:=\mathcal{I}^{h}\left(C^{l}(\bar{\Omega})\right) \subseteq C^{r}(\Omega) \cap W^{r+1, \infty}(\Omega),
$$

where $\mathcal{I}^{h}: C^{l}(\bar{\Omega}) \rightarrow L^{1}(\Omega)$ is the global interpolation operator defined by

$$
\left.\mathcal{I}^{h} v\right|_{T}:=\mathcal{I}_{T}^{h} v, \quad \text { for } T \in \mathcal{T}^{h}, h \in(0,1],
$$

and $\mathcal{I}_{T}^{h}$ is the interpolation operator for the affine-equivalent element $\left(T, \mathcal{P}_{T}, \mathcal{N}_{T}\right)$.

Let $\mathcal{P}_{k}$ be the set of polynomials in $n$ variables of degree less than or equal to $k$ with $\operatorname{dim}\left(\mathcal{P}_{k}\right)=\left(\begin{array}{c}n+k \\ k\end{array}\right)$.

Theorem 2 Let $\left\{\mathcal{T}^{h}\right\}_{h \in(0,1]}$ be a non-degenerate family of subdivisions of a polyhedral domain $\Omega$ in $\mathbb{R}^{n}, n \in \mathbb{N}$. Let $(K, \mathcal{P}, \mathcal{N})$ be a reference element satisfying

(i) $K$ is star-shaped with respect to some ball,

(ii) $\mathcal{P}_{m-1} \subseteq \mathcal{P} \subseteq W^{m, \infty}(K)$,

(iii) $\mathcal{N} \subseteq\left(C^{l}(\overline{\bar{K}})\right)^{\prime}$,

(iv) $p \in[1, \infty]$ and either $m-l-n / p>0$ when $p>1$ or $m-l-n \geqslant 0$ when $p=1$.

For all $T \in \mathcal{T}^{h}, h \in(0,1]$, let $\left(T, \mathcal{P}_{T}, \mathcal{N}_{T}\right)$ be the affine-equivalent element. Then, there exist a positive constant $C$ depending on the reference element, $n, m, p$ and the number $\rho$ in (11) such that for $0 \leqslant s \leqslant m$,

$$
\left(\sum_{T \in \mathcal{T}^{h}}\left\|v-\mathcal{I}_{T}^{h} v\right\|_{W^{s, p}(T)}^{p}\right)^{1 / p} \leqslant C h^{m-s}|v|_{W^{m, p}(\Omega)}, \quad \forall v \in W^{m, p}(\Omega) .
$$

Moreover, in the case $p=\infty$, for $0 \leqslant s \leqslant l$,

$$
\max _{T \in \mathcal{T}^{h}}\left\|v-\mathcal{I}_{T}^{h} v\right\|_{W^{s, \infty}(T)} \leqslant C h^{m-s-n / p}|v|_{W^{m, p}(\Omega)}, \quad \forall v \in W^{m, p}(\Omega) .
$$

Proof See Theorem 4.4.20 in Brenner and Scott (1996).

Theorem 3 (Inverse Estimate) Let $\left\{\mathcal{T}^{h}\right\}_{h \in(0,1]}$ be a quasi-uniform family of subdivisions of a polyhedral domain $\Omega \subseteq \mathbb{R}^{n}$. Let $(K, \mathcal{P}, \mathcal{N})$ be a reference finite element of the class $C^{r}$ such that $\mathcal{P} \subseteq W^{l, p}(K) \cap W^{m, q}(K)$ where $1 \leqslant p, q<\infty$ and $0 \leqslant m \leqslant l$. For $T \in \mathcal{T}^{h}$, let $\left(T, \mathcal{P}_{T}, \mathcal{N}_{T}\right)$ be the affine-equivalent element, and $V^{h}=\left\{v: v\right.$ is mensurable and $v_{\mid T} \in$ $\left.\mathcal{P}_{T}, \forall T \in \mathcal{P}_{T}\right\}$. Then, there exist a positive constant $C=C(l, p, q, \rho)$ such that

$$
\left[\sum_{T \in \mathcal{T}^{h}}\|v\|_{W^{l, p}(T)}^{p}\right]^{1 / p} \leqslant C h^{m-l+\min \left(0, \frac{n}{p}-\frac{n}{q}\right)}\left[\sum_{T \in \mathcal{T}^{h}}\|v\|_{W^{m, q}(T)}^{q}\right]^{1 / q}, \quad \forall v \in V^{h} .
$$


Proof See Theorem 4.5.11 in Brenner and Scott (1996).

Remark 2 In the event that the elements in the previous results form $C^{r}$ elements for some $r \geqslant 0$, then for $0 \leqslant s \leqslant r+1$, we have

$$
\begin{aligned}
& \sum_{T \in \mathcal{T}^{h}}\left\|v-\mathcal{I}_{T}^{h} v\right\|_{W^{s, p}(T)}^{p}=\left\|v-\mathcal{I}^{h} v\right\|_{W^{s, p}(\Omega)}^{p}, \\
& \max _{T \in \mathcal{T}^{h}}\left\|v-\mathcal{I}_{T}^{h} v\right\|_{W^{s, \infty}(T)}=\left\|v-\mathcal{I}^{h} v\right\|_{W^{s, \infty}(\Omega)}
\end{aligned}
$$

and

$$
\left[\sum_{T \in \mathcal{T}^{h}}\|v\|_{W^{l, \varrho}(T)}^{\varrho}\right]^{1 / \varrho}=\|v\|_{W^{s, \varrho}(\Omega)}, \quad \text { for } \varrho=p, q .
$$

Substituting these expressions in the left-hand side leads to estimates of the form

$$
\left\|v-\mathcal{I}^{h} v\right\|_{W^{s, p}(\Omega)} \leqslant C h^{m-s}|v|_{W^{m, p}(\Omega)},
$$

for all $v \in W^{m, p}(\Omega)$ and $0 \leqslant s \leqslant \min \{m, r+1\}$ and

$$
\left\|v-\mathcal{I}^{h} v\right\|_{W^{s, \infty}(\Omega)} \leqslant C h^{m-s-n / p}|v|_{W^{m, p}(\Omega)},
$$

for all $v \in W^{m, p}(\Omega)$ and $0 \leqslant s \leqslant \min \{l, r+1\}$ and

$$
\|v\|_{W^{l, p}(\Omega)} \leqslant C h^{m-l+\min \left(0, \frac{n}{p}-\frac{n}{q}\right)}\|v\|_{W^{m, q}(\Omega)},
$$

for all $v \in V^{h}$ and $0 \leqslant s \leqslant \min \{m, r+1\}$.

Now, we are able to define the discretization of the domain $\Omega$, which will allow us to obtain the proof of our main result (Theorem 1).

We assume the following hypothesis about the domain $\Omega$.

Assumption 1 Let $\Omega \subset \mathbb{R}^{n}$ be a polyhedral domain with $n \geqslant 2$ which has $\left\{\mathcal{T}^{h}\right\}_{h \in(0,1]}$ a quasi-uniform family of subdivisions with positive constant $\rho$ and let $(K, \mathcal{P}, \mathcal{N})$ be a reference element of class $C^{0}$ satisfying

(i) $K$ is star-shaped with respect to some ball,

(ii) $\mathcal{P}_{1} \subseteq \mathcal{P} \subseteq W^{2, \infty}(K)$ and

(iii) $\mathcal{N} \subseteq\left(C^{\ell}(\bar{K})\right)^{\prime}, \ell \in \mathbb{Z}_{0}^{+}$.

With this assumption and (12), we can define the finite element space

$$
X_{h}^{1 / 2}:=\left\{\mathcal{I}^{h} v: v \in C^{\ell}(\bar{\Omega}),\left.v\right|_{\partial \Omega}=0\right\} \subset X^{1 / 2} \cap C(\Omega),
$$

which has finite dimension and $\mathcal{I}^{h}$ is the global interpolation operator.

We can see that in Assumption 1, we still do not know the value of $\ell$. This value, $\ell$, comes from the inequality $m-\ell-n / p>0$ with $p \in[1, \infty]$ for $v \in W^{m, p}(\Omega)$ from Theorem 2 . In our case, $m=2=p$ and $n \geqslant 2$, then $\ell<1$. Thus, $\ell=0$.

From Assumption 1, Remark 2 and Theorems 2 and 3, there exist positive constants $C$ and $\hat{C}$ such that

$$
\begin{aligned}
\left\|v-\mathcal{I}^{h} v\right\|_{L^{2}(\Omega)} & \leqslant C h^{2}|v|_{W^{2,2}(\Omega)}, \quad \forall v \in X^{1}, \\
\left\|v-\mathcal{I}^{h} v\right\|_{W^{1,2}(\Omega)} & \leqslant C h|v|_{W^{2,2}(\Omega)}, \quad \forall v \in X^{1}, \\
\|v\|_{W^{1,2}(\Omega)} & \leqslant \hat{C} h^{-1}\|v\|_{L^{2}(\Omega)}, \quad \forall v \in X_{h}^{1 / 2} .
\end{aligned}
$$


In this framework, $A_{h}: X_{h}^{1 / 2} \rightarrow X_{h}^{1 / 2}$ given by

$$
\left\langle A_{h} \phi_{h}, \psi_{h}\right\rangle_{X}=\sigma\left(\phi_{h}, \psi_{h}\right), \quad \phi_{h}, \psi_{h} \in X_{h}^{1 / 2}
$$

is the finite element approximation of $A$. Thus, the discretization of problem (AP) can be written as (1).

Definition 8 The orthogonal projection $P_{h}: X \rightarrow X_{h}^{1 / 2}$ with relation to the inner product of $X$ is defined by

$$
\left\langle P_{h} g, v\right\rangle_{X}=\langle g, v\rangle_{X}, \quad \forall v \in X_{h}^{1 / 2} .
$$

The following result follows immediately from the above definition.

Lemma 2 The orthogonal projection $P_{h}$ satisfies

$$
\left\|P_{h} f\right\|_{X} \leqslant\|f\|_{X}, \quad \forall h \in(0,1] .
$$

Lemma 3 If (7) and (9) hold, then there is a positive constant $C$ such that

$$
\left\|P_{h} v-v\right\|_{X^{s}} \leqslant C h^{2-2 s}|v|_{H^{2}(\Omega)}, \quad \forall v \in X \text { and } s=0,1 / 2 .
$$

Proof First, we see the case $s=0$. Given $v \in X^{1}$, we obtain

$$
\left\|P_{h} v-v\right\|_{X}^{2} \leqslant\left|\left\langle P_{h} v-v, P_{h} v-v\right\rangle_{X}\right| \leqslant\left\|\chi_{h}-v\right\|_{X}\left\|P_{h} v-v\right\|_{X},
$$

where $\chi_{h}=P_{h} v \in X_{h}^{1 / 2}$. Then, $\left\|P_{h} v-v\right\|_{X} \leqslant \min _{\chi_{h} \in X_{h}^{1 / 2}}\left\|\chi_{h}-v\right\|_{X}$. Thus, using (22) and the embedding of $X^{1}$ in $X$ the result follows.

Now, let us consider the case $s=1 / 2$. Recall, the definition of the projection operator $P_{h}: X \rightarrow X^{1 / 2}$ which $A_{h} u_{h}=P_{h} v$ for some $v \in X$. Thus, from (7) and (9), we have

$$
\begin{aligned}
c_{2}\left\|P_{h} v-v\right\|_{X^{1 / 2}}^{2} & \leqslant \operatorname{Re} \sigma\left(P_{h} v-v, P_{h} v-v\right)=\operatorname{Re} \sigma\left(P_{h} v-v, v_{h}-v\right) \\
& \leqslant c_{1}\left\|P_{h} v-v\right\|_{X^{1 / 2}}\left\|v_{h}-v\right\|_{X^{1 / 2}},
\end{aligned}
$$

where $v_{h}:=A_{h} u_{h}$. Then, $\left\|P_{h} v-v\right\|_{X^{1 / 2}} \leqslant \min _{v_{h} \in X_{h}^{1 / 2}} \frac{c_{1}}{c_{2}}\left\|v_{h}-v\right\|_{X^{1 / 2}}$. Thus, using (23) we obtain

$$
\left\|P_{h} v-v\right\|_{X^{1 / 2}} \leqslant \frac{c_{1}}{c_{2}}\left\|\mathcal{I}^{h} v-v\right\|_{X^{1 / 2}} \leqslant \frac{c_{1} C}{c_{2}} h|v|_{H^{2}(\Omega)} .
$$

Since $X^{1}$ is dense in $X$, hence the result follows.

Definition 9 The map $\tilde{P}_{h}: X^{1 / 2} \rightarrow X_{h}^{1 / 2}$ defined by

$$
\sigma\left(\tilde{P}_{h} v, \chi\right)=\sigma(v, \chi), \quad \forall \chi \in X_{h}^{1 / 2}
$$

is called the elliptic projection in $X^{1 / 2}$.

Lemma 4 If (7) and (9) hold, then that

(i) The map $\tilde{P}_{h}$ is well defined, i.e., for all $v \in X^{1 / 2}$, there is a unique $v_{h} \in X_{h}^{1 / 2}$ such

$$
\sigma\left(v-v_{h}, \chi\right)=0, \quad \forall \chi \in X_{h}^{1 / 2} .
$$

(ii) There is a constant $C>0$ such that

$$
\left\|\tilde{P}_{h} v\right\|_{X^{1 / 2}} \leqslant C\|v\|_{X^{1 / 2}}, \quad \forall v \in X^{1 / 2} .
$$


Proof (i) Let $v \in X^{1 / 2}$ and define

$$
F(\chi):=\sigma(v, \chi), \quad F \in\left(X_{h}^{1 / 2}\right)^{*} .
$$

Since $\sigma$ is a continuous and coercive bilinear form in $X^{1 / 2}$ and $X_{h}^{1 / 2} \subset X^{1 / 2}$, follows that $\sigma$ is a continuous and coercive bilinear form in $X_{h}^{1 / 2}$. By Lax-Milgram (see Brenner and Scott 1996, Theorem 2.7.7), there exists a unique $v_{h} \in X_{h}^{1 / 2}$ such that

$$
\sigma\left(v_{h}, \chi\right)=F(\chi), \quad \forall \chi \in X_{h}^{1 / 2} .
$$

From (29) and (30) follows the result.

(ii) For $v \in X^{1 / 2}$, we obtain

$$
c_{2}\left\|\tilde{P}_{h} v\right\|_{X^{1 / 2}}^{2} \leqslant\left|\sigma\left(\tilde{P}_{h} v, \tilde{P}_{h} v\right)\right|=\left|\sigma\left(v, \tilde{P}_{h} v\right)\right| \leqslant c_{1}\|v\|_{X^{1 / 2}}\left\|\tilde{P}_{h} v\right\|_{X^{1 / 2}} .
$$

Therefore, $\left\|\tilde{P}_{h} v\right\|_{X^{1 / 2}} \leqslant C\|v\|_{X^{1 / 2}}$, where $C=c_{1} / c_{2}$.

Theorem 4 If Assumption 1 holds, then there exists a constant $C>0$ such that

$$
\begin{gathered}
\left\|u-\tilde{P}_{h} u\right\|_{X} \leqslant C h\|u\|_{X^{1 / 2}}, \quad u \in X^{1 / 2}, \\
\left\|u-\tilde{P}_{h} u\right\|_{X^{1 / 2}} \leqslant C h\|u\|_{X^{1}}, \quad u \in X^{1}, \\
\left\|u-\tilde{P}_{h} u\right\|_{X} \leqslant C h^{2}\|u\|_{X^{1}}, \quad u \in X^{1} .
\end{gathered}
$$

Proof We denote by $\tilde{u}^{h}:=\tilde{P}_{h} u, \hat{u}^{h}:=\mathcal{I}^{h} u$ and $e:=u-\tilde{u}^{h}$. From (27), we have

$$
\sigma\left(e, \varphi_{h}\right)=0, \quad \forall \varphi_{h} \in X_{h}^{1 / 2} .
$$

Hence, putting $\varphi_{h}=\tilde{u}^{h}-\hat{u}^{h}$, using (9), (34), (7) and (23), we obtain

$$
\|e\|_{X^{1 / 2}}^{2} \leqslant \frac{1}{c_{2}}\left|\sigma\left(e, e+\varphi_{h}\right)\right| \leqslant \frac{c_{1}}{c_{2}}\|e\|_{X^{1 / 2}}\left\|u-\hat{u}^{h}\right\|_{X^{1 / 2}} \leqslant C h\|e\|_{X^{1 / 2}}\|u\|_{H^{2}(\Omega)},
$$

which implies (32). We recall that

$$
\|e\|_{X}=\sup _{0 \neq \phi \in X} \frac{\left|(e, \phi)_{X}\right|}{\|\phi\|_{X}} .
$$

From theory of elliptic operators (see Friedman 2008), we know that given $\phi \in X$, there is a $\psi \in X^{1 / 2}$, such that

$$
\operatorname{Re} \sigma(v, \psi)=(v, \phi)_{X}, \quad \forall v \in X^{1 / 2} .
$$

Moreover, $\psi \in H^{2}(\Omega)$ and

$$
\|\psi\|_{H^{2}(\Omega)} \leqslant C\|\phi\|_{X} .
$$

Now, substituting (36) and (37) in (35), we have

$$
\|e\|_{X} \leqslant C \sup _{0 \neq \psi \in X^{1}} \frac{|\operatorname{Re} \sigma(e, \psi)|}{\|\psi\|_{H^{2}(\Omega)}} .
$$

But, again using (34), we obtain

$$
\operatorname{Re} \sigma(e, \psi)=\operatorname{Re} \sigma(e, \psi)-\operatorname{Re} \sigma\left(e, \psi_{h}\right)=\operatorname{Re} \sigma\left(e, \psi-\psi_{h}\right), \quad \forall \psi_{h} \in X_{h}^{1 / 2} .
$$

From continuity of $\sigma$ and (39), it follows

$$
|\operatorname{Re} \sigma(e, \psi)|=\left|\operatorname{Re} \sigma\left(e, \psi-\psi_{h}\right)\right| \leqslant c_{1}\|e\|_{X^{1 / 2}}\left\|\psi-\psi_{h}\right\|_{X^{1 / 2}} .
$$


Choosing $\psi_{h}$ satisfying the inequality (32), we obtain

$$
\left\|\psi-\psi_{h}\right\|_{X^{1 / 2}} \leqslant \tilde{C} h\|\psi\|_{H^{2}(\Omega)} .
$$

Then, from (38), (40) and (41), we have

$$
\|e\|_{X} \leqslant C c_{1} \sup _{0 \neq \psi \in X^{1}} \frac{\|e\|_{X^{1 / 2}} \tilde{C} h\|\psi\|_{H^{2}(\Omega)}}{\|\psi\|_{H^{2}(\Omega)}} .
$$

Thus,

$$
\|e\|_{X} \leqslant \bar{C} h\|e\|_{X^{1 / 2}} .
$$

Since $\|e\|_{X^{1 / 2}} \leqslant\|u\|_{X^{1 / 2}}$ by the definition of $\tilde{u}^{h}$ and (42), we obtain (31). Finally, replacing (32) in (42) it follows (33).

Lemma 5 If $\left\{\mathcal{T}_{h}\right\}_{h \in(0,1]}$ is quasi-uniform family of subdivisions, then there exists a positive constant $C$ such that

$$
\left\|P_{h} v\right\|_{X^{1 / 2}} \leqslant C\|v\|_{X^{1 / 2}}, \quad \forall h \in(0,1], v \in X^{1 / 2} .
$$

Proof Since $\left\|P_{h} v\right\|_{X^{1 / 2}}^{2} \leqslant\left\|P_{h} v\right\|_{X}^{2}+\left\|\nabla\left(P_{h}-I\right) v\right\|_{X}^{2}+\|\nabla v\|_{X}^{2}$, we only need to show that there exists $\bar{C}>0$ such that

$$
\left\|\nabla\left(P_{h}-I\right) v\right\|_{X} \leqslant \bar{C}\|v\|_{X^{1 / 2}}, \quad \forall v \in X^{1 / 2} .
$$

In fact, denote $\left.P_{h}\right|_{X_{h}^{1 / 2}}=I_{h}, \chi_{h}:=\tilde{P}_{h} v \in X_{h}^{1 / 2}$. Now, from (24) and (31), we obtain

$$
\begin{aligned}
\left\|\nabla\left(P_{h}-I\right) v\right\|_{X} & =\left\|\nabla\left(P_{h}-I_{h}\right)\left(v-\chi_{h}\right)\right\|_{X} \leqslant\left\|\nabla P_{h}\left(v-\chi_{h}\right)\right\|_{X}+\left\|\nabla\left(v-\chi_{h}\right)\right\|_{X} \\
& \leqslant \hat{C} h^{-1}\left\|P_{h}\left(v-\chi_{h}\right)\right\|_{X}+\|v\|_{X^{1 / 2}}+\left\|\chi_{h}\right\|_{X^{1 / 2}} \\
& \leqslant \hat{C} h^{-1}\left\|v-\chi_{h}\right\|_{X}+2\|v\|_{X^{1 / 2}} \leqslant(\hat{C}+2)\|v\|_{X^{1 / 2}},
\end{aligned}
$$

for all $v \in X^{1 / 2}$. Therefore, from Lemma 2 the result follows.

\section{Proof of the main result}

In this section, we present the proof of our main result. For this, first we will establish some results that will be needed.

Lemma 6 If (6), (7) and (9) hold, then there exists a positive constant $\delta_{1}$ such that

$$
|z|\|\varphi\|_{X}^{2}+\|\varphi\|_{X^{1 / 2}}^{2} \leqslant \delta_{1}\left|z\|\varphi\|_{X}^{2}-\sigma(\varphi, \varphi)\right|, \quad \forall \varphi \in X^{1 / 2}, \quad \forall z \in \mathcal{S}_{0, \theta_{1}} .
$$

Proof See Lemma 3.3 in Fujita and Mizutani (1976).

Lemma 7 Under the hypotheses of Lemma 6 . Given $z \in \mathcal{S}_{0, \theta_{1}}$, there is a constant $\delta_{1}>0$ such that

$$
|z|\left\|\varphi_{h}\right\|_{X}^{2}+\left\|\varphi_{h}\right\|_{X^{1 / 2}}^{2} \leqslant \delta_{1}\left|\left\langle\left(z-A_{h}\right) \varphi_{h}, \varphi_{h}\right\rangle_{X}\right|, \quad \forall \varphi_{h} \in X_{h}^{1 / 2} .
$$

Furthermore, $\mathcal{S}_{0, \theta_{1}} \subset \rho\left(A_{h}\right)$ and the following inequalities are valid

$$
\begin{gathered}
\left\|\left(z-A_{h}\right)^{-1} f_{h}\right\|_{X} \leqslant \delta_{1}\left\|f_{h}\right\|_{X} /|z|, \\
\left\|\left(z-A_{h}\right)^{-1} f_{h}\right\|_{X^{1 / 2}} \leqslant \delta_{1}\left\|f_{h}\right\|_{X} /|z|^{1 / 2}, \\
\left\|A_{h}\left(z-A_{h}\right)^{-1} f_{h}\right\|_{X} \leqslant\left(1+\delta_{1}\right)\left\|f_{h}\right\|_{X},
\end{gathered}
$$

for all $f_{h} \in X_{h}^{1 / 2}$ and $z \in \mathcal{S}_{0, \theta_{1}}$. 
Proof See Corollary 3.4 in Fujita and Mizutani (1976).

Remark 3 Given $a \in X$ and $z \in \mathcal{S}_{0, \theta_{1}}$, then

$$
\left\|A^{\alpha}(z-A)^{-1} a\right\|_{X} \leqslant C_{\alpha}|z|^{-1+\alpha}\|a\|_{X},
$$

where $C_{\alpha}=C\left(1+\delta_{1}\right)^{\alpha} \delta_{1}^{1-\alpha}$, for all $z \in \mathcal{S}_{0, \theta_{1}}, 0 \leqslant \alpha \leqslant 1$. Moreover, (49) is valid when we replace $A$ by $A_{h}$.

\section{Proof of Theorem 1}

Let $z \in \mathcal{S}_{0, \theta_{1}}$ and $f \in X$. Define $w=(z-A)^{-1} f$ and $w_{h}=\left(z-A_{h}\right)^{-1} P_{h} f$, where $w \in D(A)$ and $w_{h} \in D\left(A_{h}\right)$. From (25), $w$ and $w_{h}$ satisfy

$$
\begin{aligned}
z\langle w, \varphi\rangle_{X}-\sigma(w, \varphi) & =\langle f, \varphi\rangle_{X}, \forall \varphi \in X^{1 / 2} \text { and } \\
z\left\langle w_{h}, \varphi_{h}\right\rangle_{X}-\sigma\left(w_{h}, \varphi_{h}\right) & =\left\langle f, \varphi_{h}\right\rangle_{X}, \forall \varphi_{h} \in X_{h}^{1 / 2},
\end{aligned}
$$

, respectively. If we call $e_{h}(z)=w(z)-w_{h}(z)$ and we use the two previous equalities, we obtain

$$
z\left\langle e_{h}, \varphi_{h}\right\rangle_{X}-\sigma\left(e_{h}, \varphi_{h}\right)=0, \quad \forall \varphi_{h} \in X_{h}^{1 / 2} .
$$

Thus, given $\varphi_{h} \in X_{h}^{1 / 2}$ along with (44) and (50), we have

$$
\begin{aligned}
|z|\left\|e_{h}\right\|_{X}^{2}+\left\|e_{h}\right\|_{X^{1 / 2}}^{2} & \leqslant \delta_{1}\left|z\left\|e_{h}\right\|_{X}^{2}-\sigma\left(e_{h}, e_{h}\right)\right| \\
& =\delta_{1}\left|z\left\langle e_{h}, e_{h}+\varphi_{h}\right\rangle_{X}-\sigma\left(e_{h}, e_{h}+\varphi_{h}\right)\right| .
\end{aligned}
$$

Putting $\varphi_{h}=w_{h}-\tilde{w}^{h}$ and using (31) and (32), we obtain

$$
\begin{aligned}
|z|\left\|e_{h}\right\|_{X}^{2}+\left\|e_{h}\right\|_{X^{1 / 2}}^{2} & \leqslant \delta_{1}\left|z\left\langle e_{h}, w-\tilde{w}^{h}\right\rangle_{X}-\sigma\left(e_{h}, w-\tilde{w}^{h}\right)\right| \\
& \leqslant \delta_{1}\left(|z|\left\|e_{h}\right\|_{X}\left\|w-\tilde{w}^{h}\right\|_{X}+c_{1}\left\|e_{h}\right\|_{X^{1 / 2}}\left\|w-\tilde{w}^{h}\right\|_{X^{1 / 2}}\right) \\
& \leqslant \delta_{1} C h|z|\left\|e_{h}\right\|_{X}\|w\|_{X^{1 / 2}}+\delta_{1} c_{1} C h\left\|e_{h}\right\|_{X^{1 / 2}}\|w\|_{H^{2}(\Omega)} \\
& \leqslant C_{3} h\left(|z|\left\|e_{h}\right\|_{X}\|w\|_{X^{1 / 2}}+\left\|e_{h}\right\|_{X^{1 / 2}}\|w\|_{H^{2}(\Omega)}\right),
\end{aligned}
$$

where $C_{3}:=\max \left\{C \delta_{1}, C \delta_{1} c_{1}\right\}$. On the other hand, from (9) and (6), we have that $\operatorname{ker}(A)=0$. Thus, using the inequality (1.3.26) in Zheng (2004, p. 14) and (10), we get

$$
\|w\|_{H^{2}(\Omega)} \leqslant C_{4}\|A w\|_{X} \leqslant C_{5}\|f\|_{X}
$$

where $C_{5}:=\max \left\{C_{4}, C_{4} \delta_{1}\right\}$. Using (49) with $\alpha=1 / 2$ and (52) in (51), we have

$$
\begin{aligned}
|z|\left\|e_{h}\right\|_{X}^{2}+\left\|e_{h}\right\|_{X^{1 / 2}}^{2} & \leqslant C_{3} h\left(\delta_{1}|z|^{1 / 2}\left\|e_{h}\right\|_{X}\|f\|_{X}+C_{5}\left\|e_{h}\right\|_{X^{1 / 2}}\|f\|_{X}\right) \\
& \leqslant C_{6} h\|f\|_{X}\left(|z|^{1 / 2}\left\|e_{h}\right\|_{X}+\left\|e_{h}\right\|_{X^{1 / 2}}\right),
\end{aligned}
$$

where $C_{6}:=\max \left\{C_{3} \delta_{1}, C_{3} C_{5}\right\}$. Thus, from (53), we obtain

$$
\begin{aligned}
|z|\left\|e_{h}\right\|_{X}^{2}+\left\|e_{h}\right\|_{X^{1 / 2}}^{2} & \leqslant C_{6} h\|f\|_{X}\left(|z|^{1 / 2}\left\|e_{h}+\varphi_{h}\right\|_{X}+\left\|e_{h}+\varphi_{h}\right\|_{X^{1 / 2}}\right) \\
& \leqslant C_{6} h\|f\|_{X}\left(|z|^{1 / 2}\left\|w-\tilde{w}^{h}\right\|_{X}+\left\|w-\tilde{w}^{h}\right\|_{X^{1 / 2}}\right) \\
& \leqslant C_{6} h\|f\|_{X}\left(|z|^{1 / 2} C h\|w\|_{X^{1 / 2}}+C h\|w\|_{H^{2}(\Omega)}\right) \\
& \leqslant C_{6} C h^{2}\|f\|_{X}\left(|z|^{1 / 2}\|w\|_{X^{1 / 2}}+\|w\|_{H^{2}(\Omega)}\right) \\
& \leqslant C_{6} C h^{2}\|f\|_{X}\left(\delta_{1}\|f\|_{X}+C_{5}\|f\|_{X}\right) \leqslant C_{7} h^{2}\|f\|_{X}^{2},
\end{aligned}
$$

where $C_{7}:=\max \left\{C_{6} C \delta_{1}, C_{6} C C_{5}\right\}$. From (54), it follows (3) and (5). 
Finally, we will show (4). Let $A^{*}$ be the adjoint operator of $A . A^{*}$ is a accretive operator associated with the sesquilinear form $\sigma^{*}(u, v)=\overline{\sigma(v, u)}$ (see Henry 1981, p.203). Since $D\left(A^{*}\right)=D(A)$ for $g \in X$ and $z \in \mathcal{S}_{0, \theta_{1}}$, we define

$$
v=\left(z-A^{*}\right)^{-1} g \text { and } v_{h}=\left(z-A_{h}^{*}\right)^{-1} P_{h} g .
$$

Applying (3) and (5), and substituting $A$ and $A_{h}$ by $A^{*}$ and $A_{h}^{*}$, respectively. We have

$$
\begin{aligned}
\left|\left(e_{h}, g\right)\right| & =\left|z\left(e_{h}, v\right)-\sigma\left(e_{h}, v\right)\right|=\left|z\left(e_{h}, v-v_{h}\right)-\sigma\left(e_{h}, v-v_{h}\right)\right| \\
& \leqslant C\left(|z|\left\|e_{h}\right\|_{X}\left\|v-v_{h}\right\|_{X}+\left\|e_{h}\right\|_{X^{1 / 2}}\left\|v-v_{h}\right\|_{X^{1 / 2}}\right) \\
& \leqslant C h^{2}\left(|z|^{1 / 2}\|f\|_{X}\|v\|_{X^{1 / 2}}+\|f\|_{X}\|v\|_{H^{2}(\Omega)}\right) \\
& \leqslant C h^{2}\|f\|_{X}\|g\|_{X} .
\end{aligned}
$$

Using (35) and (55), we obtain (4).

The following remarks follow from Theorem 1 .

Remark 4 We can see that (3) and (4) hold for $z=0$.

Remark 5 From (49), the inequality of (46) and of Lemma 2, we obtain

$$
\begin{aligned}
\left\|e_{h}(z)\right\|_{X} & \leqslant\|w\|_{X}+\left\|w_{h}\right\|_{X} \leqslant \delta_{1}\|f\|_{X}|z|^{-1}+\delta_{1}\left\|P_{h} f\right\|_{X}|z|^{-1} \\
& \leqslant C_{8}\|f\|_{X}|z|^{-1} .
\end{aligned}
$$

Using (4) and (56), given $\alpha \in[0,1]$, we have

$$
\left\|e_{h}(z)\right\|_{X} \leqslant C\left\|e_{h}(z)\right\|_{X}^{\alpha}\left\|e_{h}(z)\right\|_{X}^{1-\alpha} \leqslant C_{\alpha} h^{2 \alpha}|z|^{\alpha-1}\|f\|_{X} .
$$

Remark 6 From Theorem 1, Lemma 3 and (52), we get

$$
\begin{aligned}
& \left\|\left(z-A_{h}\right)^{-1} P_{h} f-P_{h}(z-A)^{-1} f\right\|_{X^{s}} \leqslant\left\|e_{h}(z)\right\|_{X^{s}}+\left\|P_{h} w-w\right\|_{X^{s}} \\
& \quad \leqslant C h^{2-2 s}\|f\|_{X}+C h^{2-2 s}\|w\|_{H^{2}(\Omega)} \leqslant C h^{2-2 s}\|f\|_{X},
\end{aligned}
$$

for all $z \in \mathcal{S}_{0, \theta_{1}}, f \in X$ and $s=0,1 / 2$.

Acknowledgments We are grateful to the anonymous referees for a number of helpful suggestions for improvement in this article. The first author was partially supported by FAPESP (Brazil) through the research grant 09/08088-9 and the second author was partially supported by FAPESP (Brazil) through the research grant 09/08435-0.

\section{References}

Arrieta JM, Carvalho AN, Lozada-Cruz G (2006) Dynamics in dumbbell domains. I. Continuity of the set of equilibria. J Differ Equ 231(2):551-597

Brenner SC, Scott LR (1996) The mathematical theory of finite element methods. Springer-Verlag, New York Burenkov VI (1998) Sobolev spaces on domains. Teubner-texte zur mathematik (Teubner texts in mathematics), vol 137. B. G. Teubner Verlagsgesellschaft mbH, Stuttgart

Carvalho AN, Cholewa JW, Lozada-Cruz G, Primo MRT (2013) Reduction of infinite dimensional systems to finite dimensions: compact convergence approach. SIAM J Math Anal 45(2):600-638

Cholewa JW, Dlotko T (2000) Global attractors in abstract parabolic problems. In: London Mathematical Society lecture note series, vol 278. Cambridge University Press, Cambridge

Figueroa-López R, Lozada-Cruz G (2014) On global attractors for a class of parabolic problems. Appl Math Inf Sci 8(2):1-8

Friedman A (2008) Partial differential equations of parabolic type. Dover Publications Inc, Mineola 
Fujita H, Mizutani A (1976) On the finite element method for parabolic equations. I. Approximation of holomorphic semi-groups. J Math Soc Jpn 28(4):749-771

Fujita H, Saito N, Suzuki T (2001) Operator theory and numerical methods. In: Studies in mathematics and its applications, vol 30. North-Holland Publishing Co., Amsterdam

Gil' MI (2012) Norm estimates for resolvents of non-selfadjoint operators having Hilbert-Schmidt inverse ones. Math Commun 17(2):599-611

Gil' MI (2013) Resolvents of operators inverse to Schatten-von Neumann ones. Ann. Univ., Ferrara (published online December 31)

Henry D (1981) Geometric theory of semilinear parabolic equations. In: Lecture Notes in Mathematics, vol 840. Springer, New York

Pazy A (1992) Semigroups of linear operators and applications to partial differential equations. In: Applied Mathematical Sciences, 2nd edn. Springer-Verlag, New York

Zheng SM (2004) Nonlinear evolution equations. In: CRC monographs and surveys in pure and applied mathematics, vol 133. Chapman \& Hall, Boca Raton 